\title{
1 Genetic diversification of persistent Mycobacterium abscessus within Cystic 2 Fibrosis patients
}

3 Astrid Lewin $^{\mathrm{a}^{*}}$, Elisabeth Kamal ${ }^{\mathrm{a}}$, Torsten Semmler ${ }^{\mathrm{b}}$, Katja Winter ${ }^{\mathrm{c}}$, Sandra Kaiser ${ }^{\mathrm{c}}$, Hubert Schäfer ${ }^{\mathrm{a}}$,

$4 \quad$ Lei Mao ${ }^{\mathrm{a}, 1}$, Patience Eschenhagen $^{\mathrm{e}}$, Claudia Grehn $^{\mathrm{e}}$, Carsten Schwarz ${ }^{\mathrm{e}}$

5 anit 16 Mycotic and Parasitic Agents and Mycobacteria, Robert Koch Institute, Seestraße 10, Berlin, 6 Germany

7 Unit NG 1 Microbial Genomics, Robert Koch Institute, Nordufer 20, Berlin, Germany

$8{ }^{c}$ Unit MF1 Bioinformatics, Robert Koch Institute, Seestraße 10, Berlin, Germany

9 e Department of Pediatrics, Division of Pulmonology, Immunology and Intensive Care Medicine,

10 Division of Cystic Fibrosis, Charité - Universitätsmedizin, Augustenburger Platz 1, 13353 Berlin,

11 Germany

$12{ }^{1}$ present address: Unit 31 Infectious Disease Data Science Unit, Robert Koch Institute, Seestraße 10, 13 Berlin, Germany

e-mail addresses:

16 Astrid Lewin: LewinA@rki.de; Elisabeth Kamal: KamalE@ rki.de; Torsten Semmler:

17 SemmlerT@ rki.de; Katja Winter: WinterK@rki.de; Sandra Appelt: AppeltSa@ rki.de; Hubert Schäfer:

18 SchaeferH@rki.de; Lei Mao: MaoL@rki.de; Patience Eschenhagen:

19 patience.eschenhagen@charite.de; Claudia Grehn: claudia.grehn@charite.de; Carsten Schwarz:

20 Carsten.Schwarz@charite.de

*Corresponding author:

Astrid Lewin, Division 16 Mycotic and Parasitic Agents and Mycobacteria, Robert Koch Institute,

24 Seestraße 10, Berlin, Germany, Email: LewinA@rki.de

Keywords:

27 Mycobacterium abscessus; Mycobacteroides abscessus; nontuberculous mycobacteria; lung infection;

30 The authors report no conflict of interest. 

function. Treatment of these multidrug-resistant pathogens is associated with severe side-effects, while frequently unsuccessful. Insight on M. abscessus genomic evolvement during chronic lung infection would be beneficial for improving treatment strategies. A longitudinal study enrolling $42 \mathrm{CF}$ patients was performed at a CF center in Berlin, Germany, to elaborate phylogeny and genomic diversification of in-patient M. abscessus. Eleven of the $42 \mathrm{CF}$ patients were infected with M. abscessus. Global human-transmissible M. abscessus cluster strains were isolated from five of these 11 patients. Phylogenetic analysis of 88 genomes from isolates of the 11 patients excluded occurrence of $M$.

41 abscessus transmission among members of the study group. Genome sequencing and variant analysis

42 of 30 isolates from 11 serial respiratory samples collected over four years from a chronically infected 43 patient demonstrated accumulation of gene mutations. In total, 53 genes exhibiting non-synonymous 44 variations were identified. Enrichment analysis emphasized genes involved in synthesis of 45 glycopeptidolipids, genes from the embABC (arabinosyltransferase) operon, betA (glucose-methanol46 choline oxidoreductase) and choD (cholesterol oxidase). Genetic diversity evolved in a variety of 47 virulence- and resistance-associated genes. The strategy of $M$. abscessus populations in chronic lung 48 infection is not clonal expansion of dominant variants, but to sustain simultaneously a wide range of 49 genetic variants facilitating adaptation of the population to changing living conditions in the lung. 50 Genomic diversification during chronic infection requires increased attention when new control 51 strategies against M. abscessus infections are explored. 


\section{Introduction}

54 More than 30 species of nontuberculous mycobacteria (NTM) are known to cause infections in 55 humans [1], mostly in persons with immunodeficiency or underlying diseases such as Cystic Fibrosis 56 (CF) [2]. NTM prevalence in CF populations is increasing [3], which can in part be attributed to 57 medical progress resulting in extended life expectancy of CF patients. Particularly, infections with 58 Mycobacterium abscessus (MABS), also named Mycobacteroides abscessus, pose a threat to CF 59 patients as they frequently lead to lung function decrease [4] and pose a risk in lung transplantation

60 [5]. The environment has generally been assumed to be the source of NTM infection. However, recent 61 findings show that the majority of MABS in CF patients belong to three phylogenetic clusters [6]

62 suggesting human-to-human transmission as an additional potential infection route. MABS is

63 characterized by its extreme resistance towards antibiotics [7], which necessitates long-lasting 64 combination therapy. Despite such protracted therapy causing severe side effects, low culture 65 conversion rates of typically $40-50 \%$ have been reported [8]. Because of the serious side effects of the 66 antibiotic therapies, the balance between their harm and benefit for patients must be carefully weighed. 67 Although information on genomic diversity of MABS is now available [6], prognostic genomic 68 markers for disease progression have not yet been identified and knowledge on in-patient evolution of 69 MABS during chronicity is scarce. Using respiratory samples from CF patients from a German CF 70 treatment center, the focus of the present study lies on characterizing the population structure of 71 MABS in CF patients and analyzing in-patient genetic variation evolving during long-term chronic 72 infection. 


\section{Isolation and identification of NTM from Cystic Fibrosis patients}

The 42 patients involved in the study were recruited from the CF Center at the Charité-

Universitätsmedizin Berlin in Germany from 2013 to 2018. Within this period, the center treated 16

CF patients with NTM-PD. Patient characteristics are summarized in Supplementary Table S1.

Isolation of NTM was envisaged if a patient had an unclear decline in pulmonary function tests that was obtained from the ethics committee of the Charité -Universitätsmedizin Berlin (EA2/093/12).

Written consent had been obtained from all patients.

Isolation of NTM from sputum or BAL using Nalc/NaOH was performed as described in [9]. NTM colonies isolated from sputum were purified at least twice by spreading single colonies on agar plates. NTM species were determined by PCR (DreamTaq DNA Polymerase, Thermo Fisher Scientific) and sequencing (ABI BigDyeTM 3.1, Thermo Fisher Scientific) of 16S rDNA and/or ITS. Primers used are listed in Supplementary Table S2.

Cultivation of $M A B S$

MABS was grown at $37^{\circ} \mathrm{C}$ on Middlebrook $7 \mathrm{H} 11$ agar (BD Biosciences) supplemented with $10 \%$ modified ADC ( $2 \%$ glucose, $5 \%$ BSA, $0.85 \% \mathrm{NaCl})$ or in Middlebrook 7H9broth (BD Biosciences) supplemented with $10 \%$ modified ADC along with $0.05 \%$ Tween 80 without shaking.

\section{Determination of $\mathrm{MIC}$}

93 Minimal inhibitory concentrations (MIC) were determined using the Sensititre ${ }^{\mathrm{TM}}$ plates (TREK

94 Diagnostic Systems, ThermoFisher Scientific) according to the instructions of the provider. Interpretation of MIC values followed the CLSI guidelines [10], M62.

\section{Whole genome sequencing}

97 For Illumina sequencing, Paired-end (PE) DNA libraries were constructed using the Nextera XT DNA

98 kit (Illumina, San Diego, CA, USA) according to the manufacturer's protocol. The pooled library was prepared as recommended by the Illumina HiSeq v3 reagent preparation guide and loaded onto a cartridge (V3 chemistry) generating a 300 bp paired-end output. MinION one-dimensional (1D) libraries were constructed, using the SQK-RBK004 kit (Nanopore technologies, Oxford, UK), and loaded according to the manufacturer's instructions onto an R9.4 flow cell. The sequencing data was collected for $48 \mathrm{~h}$.

\section{Bioinformatic analysis}

105 Reference genomes were M. abscessus abscessus (MABSa) ATCC 19977 (NC010397.1), M. abscessus bolletii (MABSb) CIP 108541 (NZ_JRMF00000000) and M. abscessus massiliense 
(ENA project accession ERP001039] [6]. For quality control of NGS data, the in-house pipeline QCumber (v2.1.1) (https://gitlab.com/RKIBioinformaticsPipelines/QCumber) was used. QCumber employs the software tool Trimmomatic [11], which was used for Illumina adapter removal.

All draft genomes were annotated using Prokka [12]. The determination of the maximum common genome (MCG) alignment was done by identifying the genes present in all genomes [13]. Coding sequences were clustered based on the parameters sequence similarity (min. 70\%) and coverage (min. $90 \%$ ) and defined the 2,085 genes that were present in each genome while fulfilling the threshold parameters as MCG. Next the allelic variants of these genes were extracted from all genomes by a BLAST-based approach, aligned individually for each gene and then concatenated, which resulted in an alignment of $2.089 \mathrm{Mbp}$. This alignment was used to calculate a maximum likelihood-based phylogeny with RAxML v.8.2.10 with 100 bootstraps under the assumption of the gtr-gamma DNA substitution model [14]. ClonalFrameML v1.11 [15] was used to correct for recombination events. The phylogenetic tree was visualized together with the distribution of accessory genes using phandango [16].

For gene variation analysis, the genome sequences of $30 \mathrm{MABSa}$ isolates from one chronically infected patient collected in the years 2013 to 2017 were used to extract non-synonymous small nucleotide variants (nsSNV). The MinION sequence data together with Illumina data from an isolate originating from the first sample from this patient were used as a reference to identify nsSNVs in all other isolates compared to this genome sequence. To this end the MinION fast5 output files were demultiplexed with Deepbinner (v.0.2.0) [17]. Basecalling and barcode trimming was performed using Guppy (v.3.1.5) (Community.nanoporetech.com). The read quality was checked using pycoQC (v.2.3.1.2) [18], followed by (1) de-novo assembly of the initial sample, (2) reordering contigs against MABSa ATCC 19977 reference sequence, (3) mapping of the remaining samples from this patient against the final assembly, (4) gene annotation, (5) multi sample variant calling using the initial sample as starting point, (6) SNV and Indel filtering and (7) variant annotation. The assembly was performed using Unicycler (v0.4.7) [19] and reordered with progressiveMauve (v2.4.0) [20] against the MABSa reference sequence. Afterwards the remaining samples of the patient were mapped against the assembly using BWA (v0.7.15-r1140). Genes were annotated using prokka (v1.13.3). A multi sample variant calling was performed using GATK (v.4.1.2.0). SNVs and Indels were filtered according to GATKs best practice recommendations with few alterations. For SNVs the following filter was set: $\mathrm{QD}<2.0$ || FS>60.0 || ReadPosRankSum<-8.0 || MQ<40.0 || MQRankSum<-12.5 and for indels $\mathrm{QD}<2.0$ || FS $>200.0$ || ReadPosRankSum<-20.0. Variants that passed the filter were annotated with SnpEff (v4.3u) [21]. In a second filtering step only variants in coding regions were selected that

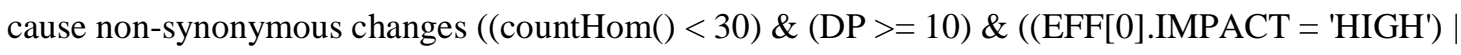
$(\mathrm{EFF}[0] . I M P A C T=$ 'MODERATE')). Sequence analysis was supported by use of Geneious software (Geneious Prime 2020, Biomatters). 
Results

\section{Predominance of MABS among isolated NTM}

147 NTM were isolated from 16 of the 42 patients (Supplementary Table S1). MABS was the most frequently isolated NTM species (11 patients) followed by M. avium hominissuis (MAH) (six patients), M. intracellulare (three patients) and M. chimaera (one patient). Two patients were coinfected by MABS and MAH, one patient by MABS and M. intracellulare, one patient by MAH and M. intracellulare and one patient by MABS, MAH and M. intracellulare.

88 isolates from the 11 patients infected with MABS were further investigated. Seven of these patients were infected with the subspecies MABSa, three with the subspecies MABSm and one patient with the subspecies MABSb. One patient had a double infection with MABSa and MABSb. 43 isolates had a rough, 43 a smooth colony morphology and two were mixed. An overview on isolates used for whole genome sequencing including subspecies, colony morphology, isolation year and accession numbers is presented in Supplementary Table S3.

\section{Absence of patient-to-patient transmission of MABS}

Illumina NGS data were used for phylogenetic analysis of all 88 isolates from 34 respiratory samples of 11 MABS-infected patients (Supplementary Table S3). Figure 1 shows a Maximum Likelihood Tree corrected for recombination events based on 2085 core genome genes identified in the genomes of the 88 isolates and the reference strains.

The principle tree structure was confirmed by a Maximum Likelihood Tree based on the pan genome (Supplementary Figure S1).

The phylogenetic tree shows the presence of well separated MABS clusters each belonging to one patient (A, B, C, H, K, S) as well as the presence of highly similar isolates belonging to different patients (E, F and D, G, O). One representative of each global MABS cluster [6] was included in the phylogenetic tree. While none of the patient isolates formed a cluster with the MABSm global cluster strain BIR948, isolates from patients $\mathrm{E}$ and F clustered with the global MABSa cluster strain RVI21, whereas isolates D, G and O clustered with global cluster strain BIR1034. Within MABSa strains up to 32 SNPs between different isolates belonging to one patient were observed in the core genome. Isolates belonging to different MABSa strains within the same global cluster exhibited SNP distances of at least 91 SNPs (cluster D/G/O/BIR1034) or at least 78 SNPs (cluster E/F/RVI21). Bryant et al. had proposed less than 20 SNPs to indicate probable recent transmission of MABS, 20-38 SNPs possible recent transmission and more than 38 SNPs no recent transmission [6]. Transmission of MABS among patients of the treatment center can thus be excluded by SNP analysis. Absence of genomes (Figure 1). 


\section{Genetic diversity evolving during persistent MABSa infection}

180 The longest tracking time and highest sample numbers were obtained for patient $\mathrm{C}$. The first

181 respiratory sample obtained from this patient was taken in the month after diagnosis of MABS

182 infection, followed by samples after five, 10, 18, 26, 32, 34, 36, 38, 49 and 53 months. Between two to

183 four colonies per sample were included in the analysis. In total, 30 isolates from 11 serial sputum

184 samples collected over 4.5 years were gained.

185 All 30 isolates were sequenced by Illumina technology (Supplementary Table S3, Figure 1 and

186 Supplementary Figure S1). The Maximum Likelihood Tree in Figure 1 shows that these isolates form

187 a cluster separated from the isolates from the other patients. One of the isolates obtained from the first

188 sample (isolate 09-13-3) was additionally sequenced by MinION technology to complete the genome

189 and provide a baseline reference for further analysis. Supplementary Table S4 summarizes the

190 statistics for genome sequence assembly of the completed genome from isolate 09-13-3. The genome

191 from isolate 09-13-3 comprised a chromosome of a size of $4.949 .160 \mathrm{bp}$ and a plasmid of $24.978 \mathrm{bp}$.

192 This plasmid (pMabs 09-13, plasmid map in Supplementary Figure S2) is similar to the plasmid from

193 strain M. sp. MS1601 (NCBI BlastN: 72\% Query cover, $97.80 \%$ identity).

194 SNV calling using the MinION genome sequence from 09-13-3 as baseline was applied to identify

195 gene variations occurring in this patient over the 4.5-years monitoring period. Only non-synonymous

196 (ns) SNVs and larger deletions as well as mutations in the rRNA genes were further considered. 53

197 genes exhibiting non-synonymous mutations with respect to isolate 09-13-3 were identified. These

198 genes and the affected isolates are listed in Table 1. 
Table 1: nsSNVs and larger deletions in isolates from serial samples obtained over 4.5 years from a chronically infected patient. Shown are the locus tags of the homologous genes in the reference strain ATCC 19977, the locus tags in the completed genome from the first sample (09-13-3), gene names, annotations, functional COG categories and names of the isolates exhibiting the mutations when compared to the initial isolate 09-13-3. Known associations to virulence and resistance of the genes or homologous genes in other mycobacterial species are indicated in the last two columns.

\begin{tabular}{|c|c|c|c|c|c|c|c|}
\hline $\begin{array}{l}\text { Locus tag in } \\
\text { ATCC } \\
19977^{1} \\
\end{array}$ & $\begin{array}{c}\text { Locus tag in } \\
09-13-3^{2}\end{array}$ & $\begin{array}{c}\text { Gene } \\
\text { name }^{3}\end{array}$ & Annotation $^{3}$ & $\begin{array}{l}\text { Functional } \\
\text { category }^{4}\end{array}$ & $\begin{array}{c}\text { Isolates exhibiting } \\
\text { SNVs }^{5}\end{array}$ & $\begin{array}{l}\text { Association of } \\
\text { gene to virulence }\end{array}$ & $\begin{array}{l}\text { Association of gene } \\
\text { to resistance }\end{array}$ \\
\hline MAB_0033c & HIFIHNKG_00044 & $p k n B$ & $\begin{array}{l}\text { Probable serine/threonine } \\
\text { kinase PknB }\end{array}$ & {$[\mathrm{K}][\mathrm{L}][\mathrm{T}]$} & $109-17-3$ & $\begin{array}{l}\text { Survival in the } \\
\text { host [22] }\end{array}$ & $\begin{array}{l}\text { B-lactam, Rifampin } \\
\text { [23] }\end{array}$ \\
\hline MAB_0173 & HIFIHNKG_00191 & & $\begin{array}{l}\text { UbiA prenyltransferse } \\
\text { family protein }\end{array}$ & {$[\mathrm{H}]$} & $65-15-13$ & & Ethambutol [24] \\
\hline MAB_0186c & HIFIHNKG_00204 & $e m b A$ & $\begin{array}{l}\text { Probable } \\
\text { arabinosyltransferase A }\end{array}$ & {$[\mathrm{M}]$} & $65-16-15$ & & Ethambutol $[24,25]$ \\
\hline MAB_0189c & HIFIHNKG_00207 & $e m b C$ & $\begin{array}{l}\text { Probable } \\
\text { arabinosyltransferase C }\end{array}$ & {$[\mathrm{M}]$} & $\begin{array}{l}30-14-1,40-14-3,40- \\
14-8,58-15-2,58-15-6, \\
58-15-9,65-16-17,65- \\
16-20,74-16-1,74-16- \\
2,74-16-3,74-16-4,77- \\
16-4,79-16-1,79-16-4, \\
79-16-6,103-17-1\end{array}$ & $\begin{array}{l}\text { LAM biosynthesis } \\
{[26]}\end{array}$ & Ethambutol [26] \\
\hline No homolog & HIFIHNKG_00238 & & Hypothetical protein & & $58-15-4$ & & \\
\hline MAB_0277c & HIFIHNKG_00346 & $t c y B$ & $\begin{array}{l}\text { Probable amino acid } \mathrm{ABC} \\
\text { transporter permease }\end{array}$ & {$[\mathrm{E}][\mathrm{P}][\mathrm{T}]$} & $65-16-20$ & & \\
\hline MAB_0310c & HIFIHNKG_00380 & & $\begin{array}{l}\text { Putative cyclopropane- } \\
\text { fatty-acyl-phospholipid } \\
\text { synthase }\end{array}$ & {$[\mathrm{M}]$} & $79-16-4$ & $\begin{array}{l}\text { Survival in the } \\
\text { host [27] }\end{array}$ & \\
\hline MAB_0315 & HIFIHNKG_00385 & $h s a A$ & $\begin{array}{l}\text { Flavin-dependent } \\
\text { monooxygenase, } \\
\text { oxygenase subunit HsaA }\end{array}$ & {$[\mathrm{I}]$} & $40-14-1$ & & \\
\hline $\begin{array}{l}\text { MAB_0415, } \\
\text { MAB_0416c }\end{array}$ & $\begin{array}{l}\text { HIFIHNKG_00492 } \\
\text { HIFIHNKG_00493 }\end{array}$ & $c r p$ & $\begin{array}{l}\text { Putative Crp/Fnr-family } \\
\text { transcriptional regulator, } \\
\text { cAMP-binding protein }\end{array}$ & $\begin{array}{l}{[\mathrm{E}][\mathrm{G}][\mathrm{K}]} \\
{[\mathrm{T}]}\end{array}$ & $\begin{array}{l}\text { 40-14-1,74_16_2,79- } \\
16-1,79-16-4,79-16-6\end{array}$ & & Clofazimine [28] \\
\hline MAB_0673 & HIFIHNKG_00715 & $t c r X$ & $\begin{array}{l}\text { Putative DNA-binding } \\
\text { response regulator PhoP }\end{array}$ & {$[\mathrm{K}][\mathrm{T}]$} & $65-16-15$ & $\begin{array}{l}\text { Iron acquisition } \\
\text { [29]; } \\
\text { Virulence [30] }\end{array}$ & \\
\hline MAB_0816 & HIFIHNKG_00859 & betA & $\begin{array}{l}\text { Probable glucose- } \\
\text { methanol-choline } \\
\text { oxidoreductase }\end{array}$ & {$[\mathrm{E}]$} & $\begin{array}{l}74-16-2,79-16-1,79- \\
16-4,79-16-6\end{array}$ & & \\
\hline MAB_0939 & HIFIHNKG_00948 & eryA & $\begin{array}{l}\text { Probable polyketide } \\
\text { synthase, Malonyl CoA- }\end{array}$ & [Q] & $40-14-3$ & Virulence $[31,32]$ & $\begin{array}{l}\text { Amikacin, } \\
\text { Cefoxitin, }\end{array}$ \\
\hline
\end{tabular}




\begin{tabular}{|c|c|c|c|c|c|c|c|}
\hline & & & $\begin{array}{l}\text { acyl carrier } \\
\text { proteintransacylase }\end{array}$ & & & & $\begin{array}{l}\text { Imipenem, } \\
\text { Linezolid [this } \\
\text { study] }\end{array}$ \\
\hline MAB_1080 & HIFIHNKG_01092 & $m s p D$ & porin & {$[\mathrm{S}]$} & $\begin{array}{l}58-15-2,103-17-3,109- \\
17-1\end{array}$ & $\begin{array}{l}\text { Iron acquisition } \\
\text { [33]; } \\
\text { Zinc acquisition } \\
\text { [34]; } \\
\text { Intracellular } \\
\text { survival [35] }\end{array}$ & $\begin{array}{l}\text { Fluoroquinolone, } \\
\text { Chloramphenicol } \\
\text { [36]; } \\
\text { Clarithromycin [37] }\end{array}$ \\
\hline MAB_1129 & HIFIHNKG_01124 & $\begin{array}{l}\text { tatD } \\
y a b D\end{array}$ & $\begin{array}{l}\text { Probable } \\
\text { deoxyribonuclease }\end{array}$ & {$[\mathrm{L}]$} & $58-15-9$ & & \\
\hline MAB_1156c & HIFIHNKG_01160 & lys $A$ & $\begin{array}{l}\text { Diaminopimelate } \\
\text { decarboxylase LysA }\end{array}$ & {$[\mathrm{E}]$} & $77-16-4$ & Virulence [38] & \\
\hline MAB_1499 & HIFIHNKG_01502 & & $\begin{array}{l}\text { Putative FAD dependent } \\
\text { oxidoreductase }\end{array}$ & {$[\mathrm{E}]$} & $74-16-3,74-16-4$ & & \\
\hline MAB_1539c & HIFIHNKG_01542 & & $\begin{array}{l}\text { Mycobacterium } \\
\text { membrane protein }\end{array}$ & {$[\mathrm{S}]$} & $103-17-1$ & & \\
\hline MAB_1607 & HIFIHNKG_01626 & $r n E$ & Possible ribonuclease E & {$[\mathrm{J}]$} & $103-17-3$ & & \\
\hline MAB_1678c & HIFIHNKG_01697 & furB & $\begin{array}{l}\text { Putative ferric uptake } \\
\text { regulator FurB }\end{array}$ & {$[\mathrm{P}]$} & $103-17-3,109-17-1$ & $\begin{array}{l}\mathrm{Zn} \text { acquisition } \\
\text { [39] }\end{array}$ & \\
\hline MAB_1881c & HIFIHNKG_01794 & & $\begin{array}{l}\text { Putative transcriptional } \\
\text { regulator, TetR family }\end{array}$ & {$[\mathrm{K}]$} & $\begin{array}{l}09-13-7,23-13-1,23- \\
13-4,30-14-1,30-14-2 \\
40-14-1,40-14-3,40- \\
14-8,58-15-2,58-15-4 \\
58-15-6,58-15-9,65- \\
16-13,65-16-15,65-16- \\
17,65-16-20,74-16-1 \\
74-16-2,74-16-3,74- \\
16-4,77-16-4,77-16-7 \\
79-16-1,79-16-4,79- \\
16-6,103-17-1,103-17- \\
3,109-17-1,109-17-3\end{array}$ & & Clarithromycin [37] \\
\hline MAB1991c & HIFIHNKG_01937 & $m p t A$ & $\begin{array}{l}\text { Integral membrane } \\
\text { protein Alpha-(1-6- } \\
\text { mannopyranosyltransferas } \\
\text { e A }\end{array}$ & [S] & $103-17-3,109-17-1$ & $\begin{array}{l}\text { LM/LAM } \\
\text { synthesis [40] }\end{array}$ & \\
\hline MAB_2161c & HIFIHNKG_02096 & & $\begin{array}{l}\text { Hypothetical low } \\
\text { molecular weight antigen } \\
\text { Mtb12 }\end{array}$ & {$[\mathrm{S}]$} & 09-13-7 & & \\
\hline MAB_2255 & HIFIHNKG_02189 & & $\begin{array}{l}\text { Probable non-ribosomal } \\
\text { peptide synthetase }\end{array}$ & [Q] & $109-17-3$ & & \\
\hline MAB_2256 & HIFIHNKG_02190 & & $\begin{array}{l}\text { Probable polyketide } \\
\text { synthase }\end{array}$ & [Q] & $103-17-3$ & & \\
\hline
\end{tabular}




\begin{tabular}{|c|c|c|c|c|c|c|c|}
\hline MAB_2299c & HIFIHNKG_02233 & & $\begin{array}{l}\text { Possible transcriptional } \\
\text { regulatory protein }\end{array}$ & {$[\mathrm{K}]$} & $79-16-4$ & & $\begin{array}{l}\text { Clofazimine, } \\
\text { Bedaquiline [28, 41] }\end{array}$ \\
\hline MAB_2570c & HIFIHNKG_02505 & $m m p L$ & $\begin{array}{l}\text { Probable membrane } \\
\text { protein, MmpL family }\end{array}$ & [D] & $65-16-20,77-16-4$ & $\begin{array}{l}\text { Transport of cell } \\
\text { wall lipids [42] }\end{array}$ & Antibiotics [42] \\
\hline MAB_2645c & HIFIHNKG_02588 & $\operatorname{trp} C$ & $\begin{array}{l}\text { Indole-3-glycerol- } \\
\text { phosphate synthase }\end{array}$ & {$[\mathrm{E}]$} & $74-16-3,74-16-4$ & $\begin{array}{l}\text { Survival in host } \\
\text { cells [43] }\end{array}$ & \\
\hline MAB_2679 & HIFIHNKG_02622 & & Uncharacterized protein & {$[\mathrm{S}]$} & $74-16-1$ & & \\
\hline MAB_2788 & HIFIHNKG_02733 & $g g t B$ & $\begin{array}{l}\text { Gamma-glutamyl- } \\
\text { transpeptidase }\end{array}$ & {$[\mathrm{E}]$} & $77-16-4$ & $\begin{array}{l}\text { Survival in host } \\
\text { cells [44] }\end{array}$ & \\
\hline MAB_2825 & HIFIHNKG_02770 & & $\begin{array}{l}\text { Acetyltransferase family } \\
\text { protein }\end{array}$ & {$[\mathrm{I}]$} & $103-17-3$ & & \\
\hline No homolog & HIFIHNKG_02868 & & Hypothetical protein & & $40-14-1$ & & \\
\hline MAB_3029 & HIFIHNKG_02972 & ideR & Iron-dependent repressor & {$[\mathrm{K}]$} & $65-16-20,77-16-4$ & $\begin{array}{l}\text { Iron acquisition } \\
\text { [45] }\end{array}$ & \\
\hline MAB_3034 & HIFIHNKG_02977 & & $\begin{array}{l}\text { Alpha/beta hydrolase fold } \\
\text { family hydrolase }\end{array}$ & {$[\mathrm{I}]$} & $65-16-20,77-16-4$ & & \\
\hline MAB_3036c & HIFIHNKG_02979 & $n r d R$ & $\begin{array}{l}\text { Transcriptional repressor } \\
\text { NrdR }\end{array}$ & {$[\mathrm{K}]$} & $40-14-3$ & & \\
\hline MAB_3404c & HIFIHNKG_03344 & $n r d F$ & $\begin{array}{l}\text { Ribonucleoside- } \\
\text { diphosphate reductase } \\
\text { subunit beta }\end{array}$ & {$[\mathrm{F}]$} & $74-16-1,103-17-1$ & & \\
\hline No homolog & HIFIHNKG_03363 & & Hypothetical protein & {$[\mathrm{S}]$} & $74-16-3$ & & \\
\hline No homolog & HIFIHNKG_03364 & & Hypothetical protein & {$[\mathrm{S}]$} & $74-16-3,74-16-4$ & & \\
\hline MAB_3623 & HIFIHNKG_03588 & & $\begin{array}{l}\text { Probable transcriptional } \\
\text { regulatory protein, AraC } \\
\text { family }\end{array}$ & {$[\mathrm{K}]$} & $65-16-20$ & & \\
\hline No homolog & HIFIHNKG_03589 & & $\begin{array}{l}\text { Hypothetical Gtr-like } \\
\text { protein }\end{array}$ & {$[\mathrm{I}][\mathrm{M}][\mathrm{S}]$} & $77-16-7,109-17-3$ & & \\
\hline MAB_3669 & HIFIHNKG_03634 & & Uncharacterized protein & {$[\mathrm{S}]$} & $40-14-8$ & & \\
\hline MAB_3698 & HIFIHNKG_03662 & & Putative $\mathrm{ABC}$ transporter & {$[\mathrm{T}][\mathrm{V}]$} & $\begin{array}{l}40-14-3,40-14-8,58- \\
15-2,74-16-2,79-16-1, \\
79-16-4,79-16-6\end{array}$ & & \\
\hline MAB_3719c & HIFIHNKG_03684 & choD & $\begin{array}{l}\text { Putative cholesterol } \\
\text { oxidase ChoD }\end{array}$ & {$[\mathrm{E}]$} & $103-17-3,109-17-1$ & $\begin{array}{l}\text { Survival in host } \\
\text { cells [46] }\end{array}$ & \\
\hline MAB_4098c & HIFIHNKG_04080 & $\begin{array}{l}\text { mps2, } \\
\lg r D \_2\end{array}$ & $\begin{array}{l}\text { Probable peptide } \\
\text { synthetase NRP }\end{array}$ & [Q] & $\begin{array}{l}58-15-4,103-17-3,109- \\
17-1\end{array}$ & Virulence $[32,47]$ & $\begin{array}{l}\text { Amikacin, } \\
\text { Cefoxitin, } \\
\text { Imipenem, } \\
\text { Linezolid [this } \\
\text { study] }\end{array}$ \\
\hline MAB_4099c & HIFIHKG_04081 & $\begin{array}{l}\text { mpsl, } \\
\lg C_{-} 1\end{array}$ & $\begin{array}{l}\text { Probable non-ribosomal } \\
\text { peptide synthetase }\end{array}$ & [Q] & $\begin{array}{l}58-15-2,65-16-17,65- \\
16-20,74-16-3,74-16- \\
4,79-16-1\end{array}$ & Virulence $[32,47]$ & $\begin{array}{l}\text { Amikacin, } \\
\text { Cefoxitin, } \\
\text { Imipenem, }\end{array}$ \\
\hline
\end{tabular}




\begin{tabular}{|c|c|c|c|c|c|c|c|}
\hline & & & & & & & $\begin{array}{l}\text { Linezolid [this } \\
\text { study]; } \\
\text { Clofazimine [28] }\end{array}$ \\
\hline MAB_4103c & HIFIHNKG_04086 & & $\begin{array}{l}\text { Probable } \\
\text { methyltransferase }\end{array}$ & [Q] & $74-16-3,74-16-4$ & Virulence [48] & \\
\hline MAB_4105c & HIFIHNKG_04088 & $m t f D$ & Methyltransferase & [E] [S] & $74-16-3,74-16-4$ & & \\
\hline MAB_4320c & HIFIHNKG_04305 & & $\begin{array}{l}\text { Putative TetR } \\
\text { transcriptional regulator }\end{array}$ & {$[\mathrm{K}]$} & $103-17-3$ & & \\
\hline MAB_4372 & HIFIHNKG_04358 & & $\begin{array}{l}\text { Putative } 3-(2,3- \\
\text { dihydroxyphenyl) } \\
\text { propionic acid } \\
\text { dioxygenase }\end{array}$ & {$[\mathrm{S}]$} & $58-15-2$ & & \\
\hline MAB_4654 & HIFIHNKG_04625 & $\operatorname{mau} C$ & $\begin{array}{l}\text { Conserved hypothetical } \\
\text { protein (plastocyanin- } \\
\text { like) }\end{array}$ & {$[\mathrm{C}]$} & $74-16-3,74-16-4$ & & \\
\hline MAB_4690c & HFIHNKG_04663 & $\lg r C_{-} 2$ & $\begin{array}{l}\text { Probable non-ribosomal } \\
\text { peptide synthetase PstA, } \\
\text { Linear gramicidin } \\
\text { synthase subunit C }\end{array}$ & [Q] & $65-16-17$ & & \\
\hline MAB_4695c & HIFIHNKG_04668 & & $\begin{array}{l}\text { Putative } \\
\text { glycosyltransferase/rhamn } \\
\text { osyltransferase }\end{array}$ & [C] [G] & $65-16-20$ & & \\
\hline MAB_4760 & HIFIHNKG_04733 & nox & Probable nitroreductase & {$[\mathrm{C}]$} & $109-17-3$ & & \\
\hline MAB_r5052 & & $r r l$ & 23S rRNA & & $103-17-3,109-17-1$ & & Clarithromycin [49] \\
\hline
\end{tabular}

${ }^{1}$ accession: NC010397.1; ${ }^{2}$ accession: ERS4791737; ${ }^{3}$ according to NCBI (https://www.ncbi.nlm.nih.gov/), STRING (https://string-db.org/), Uniprot (https://www.uniprot.org/); ${ }^{4}$ according to EggNOG (http://eggnog5.embl.de/\#/app/seqscan). ${ }^{5}$ See Supplementary Table S3 for details of isolates. COG categories are: [C] Energy production and conversion, [D] Cell cycle control, cell division, chromosome partitioning, [E] Amino acid transport and metabolism, [F] Nucleotide transport and metabolism, [G] Carbohydrate transport and metabolism, [H] Coenzyme transport and metabolism, [I] Lipid transport and metabolism, [J] Translation, ribosomal structure and biogenesis, [K] Transcription, [L] Replication, recombination and repair, [M] Cell wall/membrane/envelope biogenesis, [P] Inorganic ion transport and metabolism, [Q] Secondary metabolites biosynthesis, transport and catabolism, [S] Function unknown, [T] Signal transduction mechanisms, [V] Defense mechanisms. nsSNV: non-synonymous small nucleotide variation. 
210 A visualization of the frequency and chronology of gene mutations occurring in the 30 isolates is

211 provided in Figure 2. The number of mutations increased from 18 months after MABS diagnosis.

212 Occurrence of dominating clones exhibiting and sustaining specific combinations of mutations was not

213 observed during the observation time period. However, a clear trend was observed with respect to the

214 loss of the plasmid pMabs over time. The strain 09-13-3 originally contained a plasmid of a size of

$21524.978 \mathrm{bp}$ (Supplementary Figure S2). This plasmid was lost from the MABS strain in the course of

216 chronic infection. Specifically, all isolates contained the plasmid until ten months after MABS

217 diagnosis. However, it remained absent all in isolates sampled at and after 38 months (Figure 2).

218 Network analysis with the set of mutated genes by STRING (https://string-db.org/) identified ten

219 functionally enriched PFAM protein domains (Table 2).

220

Table 2: Enrichment of PFAM protein domains in the pool of genes displaying non-synonymous mutations in the M. abscessus isolates from patient $\mathrm{C}$ according to String network analysis.

\begin{tabular}{|c|c|c|}
\hline $\begin{array}{l}\text { PFAM Protein domain and term } \\
\text { description }\end{array}$ & Matching proteins ${ }^{1}$ & False discovery rate \\
\hline $\begin{array}{l}\text { PF00550 } \\
\text { Phosphopantetheine attachment site }\end{array}$ & $\begin{array}{l}\text { MAB_0939(eryA), MAB_2255, } \\
\text { MAB_2256, MAB_4690c }\left(l g r \_C 2\right), \\
\text { MAB_4099c (lgrC_l), MAB_4098c } \\
(\text { lgrD_2) }\end{array}$ & $7.23 \times 10^{-5}$ \\
\hline $\begin{array}{l}\text { PF00668 } \\
\text { Condensation domain }\end{array}$ & $\begin{array}{l}\text { MAB_2255, MAB_4098c }\left(\operatorname{lgr} D \_2\right), \\
\text { MAB_4099c }\left(l g r C \_1\right), \text { MAB_4690c } \\
\left(l g r \_C 2\right.\end{array}$ & 0.00093 \\
\hline $\begin{array}{l}\text { PF13193 } \\
\text { AMP-binding enzyme C-terminal } \\
\text { domain }\end{array}$ & $\begin{array}{l}\text { MAB_2255, MAB_4098c }\left(\lg r D \_2\right) \text {, } \\
\text { MAB_4099c }\left(\lg r C_{-} l\right), \text { MAB_4690c } \\
\left(l g r \_C 2\right)\end{array}$ & 0.0153 \\
\hline $\begin{array}{l}\mathrm{PF} 04602 \\
\text { Mycobacterial cell wall arabinan } \\
\text { synthesis protein }\end{array}$ & $\begin{array}{l}\text { MAB_0186c (embA), MAB_0189c } \\
(e m b C)\end{array}$ & 0.0164 \\
\hline $\begin{array}{l}\text { PF14896 } \\
\text { EmbC C-terminal domain }\end{array}$ & $\begin{array}{l}\text { MAB_0186c (embA), MAB_0189c } \\
(e m b \bar{C})\end{array}$ & 0.0164 \\
\hline $\begin{array}{l}\text { PF00732 } \\
\text { GMC oxidoreductase }\end{array}$ & MAB_0816 (betA_l), MAB_3719c (choD) & 0.0227 \\
\hline $\begin{array}{l}\text { PF05199 } \\
\text { GMC oxidoreductase }\end{array}$ & MAB_0816 (betA_l), MAB_3719c (choD) & 0.0227 \\
\hline $\begin{array}{l}\text { PF00501 } \\
\text { AMP-binding enzyme }\end{array}$ & $\begin{array}{l}\text { MAB_2255, MAB_4098c }\left(\lg r D \_2\right), \\
\text { MAB_4099c }\left(l g r C \_1\right), \text { MAB_4690c } \\
\left(l g r \_C 2\right)\end{array}$ & 0.0264 \\
\hline $\begin{array}{l}\text { PF16197 } \\
\text { Ketoacyl-synthetase C-terminal } \\
\text { extension }\end{array}$ & MAB_0939 (eryA), MAB_2256 & 0.0264 \\
\hline $\begin{array}{l}\text { PF00698 } \\
\text { Acyl transferase domain }\end{array}$ & MAB_0939 (eryA), MAB_2256 & 0.0488 \\
\hline
\end{tabular}

${ }^{1}$ Locus tags (gene names) of the homologs in the reference strain ATCC 19977. 

was among the functionally enriched proteins as well as MAB_2255 and MAB_2256, which also encode a probable non-ribosomal peptide synthetase and a probable polyketide synthase. Furthermore, betA (probable glucose-methanol-choline oxidoreductase) and choD (putative cholesterol oxidase) were among enriched genes.

The mutations in the GPL synthesis genes [50] can explain the rough morphotype of all rough isolates. thin layer chromatography (TLC) with GPL extracted from a smooth and four rough isolates exhibiting different types of mutations (Supplementary Figure S3).

Comparison of MICs from nine rough and nine smooth paired isolates originating from the same sputum samples showed different median MICs for Amikacin, Cefoxitin, Imipenem and Linezolid. Interestingly, rough colonies showed higher median MIC values to three of these antibiotics (Amikacin, Cefoxitin and Imipenem), while they exhibited a lower median MIC to one of them (Linezolid) (Figure 3).

\section{Diversification in virulence- and resistance associated genes during chronic lung infection} homologs in other mycobacteria were published to be involved in mycobacterial virulence (Table 1). These genes or their homologs exert an impact (i) on survival in the host or host cells [MAB_0033c $(p k n B)$, MAB_0310c, MAB_1080 (porin), MAB_2645c (trpC), MAB_2788 (ggtB) MAB_3719c $(c h o D)]$, (ii) on cell wall synthesis [MAB_0189c (embC), MAB_1991c (mptA), MAB_2570c $(m m p L)],($ iii) on iron and zinc acquisition [MAB_0673 (tcrX), MAB_1080 (porin), MAB_1678c (furB), MAB_3029 (ideR)], and (iv) on GPL synthesis [MAB_0939 (eryA), MAB_4098c (mps2), MAB_4099c (mps1)]. References are provided in Table 1.

Thirteen of the 53 MABS genes or their homologs in other mycobacterial species are known to be related to antibiotic resistance (Table 1). It has been shown that genes or homologs in other mycobacteria to MAB_0173, MAB_0186c (embA), and 0189c (embC) are related to Ethambutol resistance. Clofazimine resistance was associated to genes MAB_0146c (crp), MAB_2299c, and MAB_4099c (mps1). MAB_2299c had additionally been associated with Bedaquiline resistance. Mycobacterial porin genes such as MAB_1080 were related to resistance towards, Fluoroquinolone,

256 Chloramphenicol and Clarithromycin. Clarithromycin resistance may additionally be influenced by 257 gene MAB_1881c and $r r l$. The mutation that was identified in the $r r l$ gene (position 2270, A/C) is 258 known to confer acquired Clarithromycin resistance. PknB from mycobacteria was shown to impact resistance towards $ß$-lactams and Rifampin. References are provided in Table 1. 
Discussion

261 MABS is a highly problematic multi-drug resistant pathogen. Despite protracted combination therapy

262 accompanied by severe side effects, only low conversion rates of typically 40-50\% are reported [8]

263 calling for more personalized treatment also consideration of the mycobacterial population dynamics.

264 Accordingly, the present study focused on exploring the strategy of chronic MABS to adapt to the

265 lung environment in CF patients.

266 MABS predominated NTM infections in our study group, followed by MAH. The MABS subspecies

267 distribution with $63.6 \%$ of subspecies abscessus, $27.3 \%$ of subspecies massiliense and $9 \%$ of

268 subspecies bolletii was highly similar to the distribution found in the global study from Bryant et al

$269[6]$.

270 Five of the eleven MABS-infected patients carried strains belonging to one of three global human

271 transmissible clusters, that have been reported to be more virulent and resistant and at the same time

272 more frequently associated with chronic disease compared to sporadic strains [6]. Nevertheless, the

273 results from the SNP analysis ruled out transmission of MABS between patients in the study group.

274 This was additionally supported by comparison of the accessory genomes of the isolates. Comparison

275 of accessory genomes for discrimination of closely related MABS isolates was also proposed by

276 Davidson [51]. Other studies (e.g. [52, 53]) had also reported the presence of global cluster strains in

277 patient cohorts without evidence for transmission among patients. The absence of patient-to-patient

278 transmission endorses infection control measures in the CF center in Berlin which involve among

279 others spatial and/or temporal separation of patients with NTM in respiratory tract and a face mask

280 wearing during the entire clinical stay [54].

281 Comparative genome analysis of 30 isolates from 11 serial samples from a chronically infected patient

282 identified 53 genes with non-synonymous variations. Additionally, the plasmid from this strain present

283 in the beginning was lost during persistent infection. This may be explained by the fitness cost of

284 plasmid maintenance in stress conditions present in the human host and is in good accordance to a

285 study by Shoulah et al. [55], who found more plasmid-derived genes in environmental compared to

286 clinical isolates from MAH.

287 Isolates with mutations in these 53 genes are extremely valuable to study virulence and resistance

288 mechanisms of persistent MABS, which are currently insufficiently investigated. Frequent genetic

289 changes included those leading to GPL deficiency and rough colony morphotype. These mutations

290 were associated with increased MICs for Amikacin, Cefoxitin and Imipenem and decreased MIC for

291 Linezolid. In contrast to the present study comparing MICs of isogenic isolates, previous studies on

292 strains isolated from different patients let to controversial outcomes [56, 57]. Our study emphasizes

293 the need to give more attention to the impact of morphotypes on drug resistance when searching for

294 new anti-mycobacterial drugs. 
Of the 53 genes exhibiting genetic diversity, at least 23 genes or their homologs in other mycobacteria have been assigned to be virulence- and/or resistance-associated. Genetic diversity evolved in genes related to resistance to Ethambutol, Rifampin, Clofazimine, Bedaquiline, Fluoroquinolone, Chloramphenicol, Imipenem, Cefoxitin and Clarithromycin. Two isolates had acquired the A to C mutation at position 2270 (MABS numbering) in the $r r l$ gene, a mutation known to confer acquired Clarithromycin resistance [58].

Interestingly, in-patient evolution did not bring forth fewer dominating sub-populations but rather fostered the co-existence of diverse mutant sub-populations. The upsurge of genetic diversity possibly enables the population to adapt to changing living conditions as illustrated by the following example.

MAB ecology combines the ability to survive both in the environment and in human airways, which offer disparate access to biometals such as zinc and iron. Such metals are, on the one hand, essential co-factors of enzymes and structural components of regulatory proteins. On the other hand, however, excessive concentrations thereof can be toxic. Therefore, a stringent regulatory system for homeostasis is required. The sputum from CF patients displays highly enriched metal concentrations of zinc and iron [59], which may favor genetic diversity within genes involved in zinc and iron homeostasis during chronic infection. Zinc uptake in mycobacteria is regulated by zur-smtB. Among the genes exhibiting gene diversity in persistent infection, zur is a zinc-binding repressor controlling genes involved in zinc uptake [34]. The porin MspD was shown to be induced by zinc starvation or zur deletion [34]. Moreover, MAB_1080, which was annotated as the porin protein MspD, was also among the genes exhibiting diversity upon chronic infection. Mutations were also identified in ideR, a gene that regulates transcription in response to iron levels [60], as well as in $t c r X$, which was shown to be up-regulated when M. tuberculosis was grown under iron-limited conditions [29]. Furthermore, Msp porins of rapid growing mycobacteria promote growth in nutrient-limited conditions by enhancing diffusion of small hydrophilic molecules into the cells. At the same time, however, they limit intracellular survival by increasing vulnerability to killing by reactive nitrogen [35, 61]. Therefore, the mutation in MAB_1080 may additionally impact the survival of MABS in macrophages.

322 ChoD, a cholesterol oxidase, is needed in M. tuberculosis survival in macrophages [46]. A mutant 323 deficient in the gamma-glutamyl-transpeptidase (GgtB) from M. tuberculosis, which is homologous to MAB_2788, was shown to be resistant to the toxic effects of Glutathione/ S-nitrosoglutathione and therefore better survived in macrophages [44]. Macrophages are not the only host cells for mycobacteria, some of which are also able to replicate in Type II alveolar epithelial cells (AECs). The

327 tryptophan synthesis gene $\operatorname{trpC}$ from $M$. tuberculosis is strongly up-regulated during growth in AECs

328 [43] and also the homologue from this gene was mutated in two of the MABS isolates. 
329 MptA, EmbC and MmpL family proteins are involved in lipid/glycolipid synthesis. MptA is a 330 mannosyltransferase necessary for synthesis of the mannan backbone from Lipomannan. EmbC from

331 M. tuberculosis catalyses arabinosylation of Lipomannan to form Lipoarabinomannan (LAM), which

332 is involved in immune response by interacting with TLR2 and mannose-receptor [26, 40, 62].

333 Interestingly, a longitudinal analysis by Kreutzfeld [63] of 6 MABS bolletii isolates from a CF patient

334 collected over 11 years and also identified mutations in the embABC operon during chronic infection.

335 In conclusion, our data indicate that the survival strategy of MABS in the CF lung is not towards the

336 clonal expansion of few dominant variants but the preservation of heterogeneous subpopulations

337 allowing adaptation to changing lung conditions. Similar future longitudinal studies involving other

338 MABS strains and patients will further explore the range of variation of MABS in-patient evolution

339 during chronic lung infection. Our study suggests that intervention procedures against MABS should

340 target MABS populations instead of specific isolates. 


\section{Acknowledgements}

343 We would like to thank the patients with CF, their families and the team of the CF center in Berlin.

344 We also thank Andrea Thürmer (Robert Koch Institute Berlin) and her team for genome sequencing

345 and Nils Höpner (Robert Koch Institute Berlin) for experimental support. The work from Carsten

346 Schwarz was supported by a German Infectiology Award.

347 Funding:

348 The work from Carsten Schwarz was supported by a German Infectiology Award.

349 Disclosure of interest:

350 The authors report no conflict of interest.

351 Data Availability Statement

352 All genome sequences have been submitted to the European Nucleotide Archive (ENA).

353 Data deposition:

354 Genome sequences are deposited at the European Nucleotide Archive (ENA).

\section{Ethical statement:}

356 Permission for the study was obtained from the ethics committee of the Charité -Universitätsmedizin

357 Berlin (EA2/093/12). Written consent had been obtained from all patients.

358 Statement to health and safety regulations:

359 The authors confirm compliance with national health and safety regulations. 
References

1. Heifets L: Mycobacterial infections caused by nontuberculous mycobacteria. Seminars in

2. Johnson MM, Odell JA: Nontuberculous mycobacterial pulmonary infections. Journal of thoracic disease 2014, 6(3):210-220.

3. Martiniano SL, Nick JA, Daley CL: Nontuberculous Mycobacterial Infections in Cystic Fibrosis. Clin Chest Med 2016, 37(1):83-96.

4. Esther CR, Jr., Esserman DA, Gilligan P, Kerr A, Noone PG: Chronic Mycobacterium abscessus infection and lung function decline in cystic fibrosis. Journal of cystic fibrosis : official journal of the European Cystic Fibrosis Society 2010, 9(2):117-123.

5. Lynch JP, 3rd, Sayah DM, Belperio JA, Weigt SS: Lung transplantation for cystic fibrosis: results, indications, complications, and controversies. Semin Respir Crit Care Med 2015, 36(2):299-320.

6. Bryant JM, Grogono DM, Rodriguez-Rincon D, Everall I, Brown KP, Moreno P, Verma D, Hill E, Drijkoningen J, Gilligan P et al: Emergence and spread of a human-transmissible multidrugresistant nontuberculous mycobacterium. Science 2016, 354(6313):751-757.

7. Nessar R, Cambau E, Reyrat JM, Murray A, Gicquel B: Mycobacterium abscessus: a new antibiotic nightmare. J Antimicrob Chemother 2012, 67(4):810-818.

8. Martiniano SL, Davidson RM, Nick JA: Nontuberculous mycobacteria in cystic fibrosis: Updates and the path forward. Pediatr Pulmonol 2017, 52(S48):S29-S36.

9. Ruangkiattikul N, Rys D, Abdissa K, Rohde M, Semmler T, Tegtmeyer PK, Kalinke U, Schwarz C, Lewin A, Goethe R: Type I interferon induced by TLR2-TLR4-MyD88-TRIF-IRF3 controls Mycobacterium abscessus subsp. abscessus persistence in murine macrophages via nitric oxide. Int J Med Microbio/ 2019, 309(5):307-318.

10. Woods GL B-EB, Conville PS, et al.: Susceptibility Testing of Mycobacteria, Nocardiae, and Other Aerobic Actinomycetes. Clinical and Laboratory Standards Institute 2011, 2nd edition.

11. Bolger AM, Lohse M, Usadel B: Trimmomatic: a flexible trimmer for Illumina sequence data. Bioinformatics 2014, 30(15):2114-2120.

12. Seemann T: Prokka: rapid prokaryotic genome annotation. Bioinformatics 2014, 30(14):2068-2069.

13. von Mentzer A, Connor TR, Wieler LH, Semmler T, Iguchi A, Thomson NR, Rasko DA, Joffre E, Corander J, Pickard D et al: Identification of enterotoxigenic Escherichia coli (ETEC) clades with long-term global distribution. Nat Genet 2014, 46(12):1321-1326.

14. Stamatakis A: RAxML version 8: a tool for phylogenetic analysis and post-analysis of large phylogenies. Bioinformatics 2014, 30(9):1312-1313.

15. Didelot $X$, Wilson DJ: ClonalFrameML: efficient inference of recombination in whole bacterial genomes. PLoS Comput Biol 2015, 11(2):e1004041.

16. Hadfield J, Croucher NJ, Goater RJ, Abudahab K, Aanensen DM, Harris SR: Phandango: an interactive viewer for bacterial population genomics. Bioinformatics 2018, 34(2):292-293.

17. Wick RR, Judd LM, Holt KE: Deepbinner: Demultiplexing barcoded Oxford Nanopore reads with deep convolutional neural networks. PLOS Comput Biol 2018, 14(11):e1006583.

18. Leger A, Leonardi T: pycoQC, interactive quality control for Oxford Nanopore Sequencing. Journal of Open Source Software 2019, 4(34):1236.

19. Wick RR, Judd LM, Gorrie CL, Holt KE: Unicycler: Resolving bacterial genome assemblies from short and long sequencing reads. PLoS Comput Biol 2017, 13(6):e1005595.

20. Darling AE, Mau B, Perna NT: progressive Mauve: multiple genome alignment with gene gain, loss and rearrangement. PLOS One 2010, 5(6):e11147.

21. Cingolani P, Platts A, Wang le L, Coon M, Nguyen T, Wang L, Land SJ, Lu X, Ruden DM: A program for annotating and predicting the effects of single nucleotide polymorphisms, 
SnpEff: SNPs in the genome of Drosophila melanogaster strain w1118; iso-2; iso-3. Fly 2012, 6(2):80-92.

22. Chawla Y, Upadhyay S, Khan S, Nagarajan SN, Forti F, Nandicoori VK: Protein kinase B (PknB) of Mycobacterium tuberculosis is essential for growth of the pathogen in vitro as well as for survival within the host. J Biol Chem 2014, 289(20):13858-13875.

23. Zeng J, Platig J, Cheng TY, Ahmed S, Skaf Y, Potluri LP, Schwartz D, Steen H, Moody DB, Husson RN: Protein kinases PknA and PknB independently and coordinately regulate essential Mycobacterium tuberculosis physiologies and antimicrobial susceptibility. PLOS Pathog 2020, 16(4):e1008452.

24. Giri A, Gupta S, Safi H, Narang A, Shrivastava K, Kumar Sharma N, Lingaraju S, Hanif M, Bhatnagar A, Menon B et al: Polymorphisms in Rv3806c (ubiA) and the upstream region of embA in relation to ethambutol resistance in clinical isolates of Mycobacterium tuberculosis from North India. Tuberculosis (Edinb) 2018, 108:41-46.

25. Li MC, Chen R, Lin SQ, Lu Y, Liu HC, Li GL, Liu ZG, Zhao XQ, Zhao LL, Wan KL: Detecting Ethambutol Resistance in Mycobacterium tuberculosis Isolates in China: A Comparison Between Phenotypic Drug Susceptibility Testing Methods and DNA Sequencing of embAB. Front Microbiol 2020, 11:781.

26. Korkegian A, Roberts DM, Blair R, Parish T: Mutations in the essential arabinosyltransferase EmbC lead to alterations in Mycobacterium tuberculosis lipoarabinomannan. $J$ Biol Chem 2014, 289(51):35172-35181.

27. Mendum TA, Chandran A, Williams K, Vordermeier HM, Villarreal-Ramos B, Wu H, Singh A, Smith AA, Butler RE, Prasad A et al: Transposon libraries identify novel Mycobacterium bovis BCG genes involved in the dynamic interactions required for BCG to persist during in vivo passage in cattle. BMC Genomics 2019, 20(1):431.

28. Chen Y, Chen J, Zhang S, Shi W, Zhang W, Zhu M, Zhang Y: Novel Mutations Associated with Clofazimine Resistance in Mycobacterium abscessus. Antimicrob Agents Chemother 2018, 62(7).

29. Bacon J, Dover LG, Hatch KA, Zhang Y, Gomes JM, Kendall S, Wernisch L, Stoker NG, Butcher $\mathrm{PD}$, Besra GS et al: Lipid composition and transcriptional response of Mycobacterium tuberculosis grown under iron-limitation in continuous culture: identification of a novel wax ester. Microbiology 2007, 153(Pt 5):1435-1444.

30. Parish T, Smith DA, Kendall S, Casali N, Bancroft GJ, Stoker NG: Deletion of two-component regulatory systems increases the virulence of Mycobacterium tuberculosis. Infect Immun 2003, 71(3):1134-1140.

31. Gutierrez AV, Viljoen A, Ghigo E, Herrmann JL, Kremer L: Glycopeptidolipids, a Double-Edged Sword of the Mycobacterium abscessus Complex. Front Microbiol 2018, 9:1145.

32. Catherinot E, Clarissou J, Etienne G, Ripoll F, Emile JF, Daffe M, Perronne C, Soudais C, Gaillard JL, Rottman M: Hypervirulence of a rough variant of the Mycobacterium abscessus type strain. Infect Immun 2007, 75(2):1055-1058.

33. Jones $\mathrm{CM}$, Niederweis $\mathrm{M}$ : Role of porins in iron uptake by Mycobacterium smegmatis. J Bacteriol 2010, 192(24):6411-6417.

34. Goethe E, Laarmann K, Lührs J, Jarek M, Meens J, Lewin A, Goethe R: Critical Role of Zur and SmtB in Zinc Homeostasis of Mycobacterium smegmatis. mSystems 2020, 5(2).

35. Sharbati-Tehrani S, Stephan J, Holland G, Appel B, Niederweis M, Lewin A: Porins limit the intracellular persistence of Mycobacterium smegmatis. Microbiology 2005, 151(Pt 7):24032410.

36. Danilchanka O, Pavlenok M, Niederweis M: Role of porins for uptake of antibiotics by Mycobacterium smegmatis. Antimicrob Agents Chemother 2008, 52(9):3127-3134.

37. Li B, Guo Q, Mao Y, Zou Y, Zhang Y, Zhang Z, Chu H: Genetic Evolution of Mycobacterium abscessus Conferring Clarithromycin Resistance during Long-Term Antibiotic Therapy. Can Respir J 2020, 2020:7623828. 
462 38. Shea JE, Santangelo JD, Feldman RG: Signature-tagged mutagenesis in the identification of

39. Lucarelli D, Russo S, Garman E, Milano A, Meyer-Klaucke W, Pohl E: Crystal structure and function of the zinc uptake regulator FurB from Mycobacterium tuberculosis. $J$ Biol Chem 2007, $282(13): 9914-9922$.

40. Umesiri FE, Sanki AK, Boucau J, Ronning DR, Sucheck SJ: Recent advances toward the inhibition of mAG and LAM synthesis in Mycobacterium tuberculosis. Medicinal research reviews 2010, 30(2):290-326.

41. Richard M, Gutierrez AV, Viljoen A, Rodriguez-Rincon D, Roquet-Baneres F, Blaise M, Everall I, Parkhill J, Floto RA, Kremer L: Mutations in the MAB_2299c TetR Regulator Confer CrossResistance to Clofazimine and Bedaquiline in Mycobacterium abscessus. Antimicrob Agents Chemother 2019, 63(1).

42. Bailo R, Bhatt A, Ainsa JA: Lipid transport in Mycobacterium tuberculosis and its implications in virulence and drug development. Biochem Pharmacol 2015, 96(3):159-167.

43. Ryndak MB, Singh KK, Peng Z, Laal S: Transcriptional profile of Mycobacterium tuberculosis replicating in type II alveolar epithelial cells. PLoS One 2015, 10(4):e0123745.

44. Dayaram YK, Talaue MT, Connell ND, Venketaraman V: Characterization of a glutathione metabolic mutant of Mycobacterium tuberculosis and its resistance to glutathione and nitrosoglutathione. J Bacteriol 2006, 188(4):1364-1372.

45. Pandey SD, Choudhury M, Yousuf S, Wheeler PR, Gordon SV, Ranjan A, Sritharan M: Ironregulated protein HupB of Mycobacterium tuberculosis positively regulates siderophore biosynthesis and is essential for growth in macrophages. J Bacteriol 2014, 196(10):18531865.

46. Brzostek A, Dziadek B, Rumijowska-Galewicz A, Pawelczyk J, Dziadek J: Cholesterol oxidase is required for virulence of Mycobacterium tuberculosis. FEMS Microbiol Lett 2007, 275(1):106-112.

47. Pawlik A, Garnier G, Orgeur M, Tong P, Lohan A, Le Chevalier F, Sapriel G, Roux AL, Conlon K, Honore $\mathrm{N}$ et al: Identification and characterization of the genetic changes responsible for the characteristic smooth-to-rough morphotype alterations of clinically persistent Mycobacterium abscessus. Mol Microbiol 2013, 90(3):612-629.

48. Pang L, Tian X, Pan W, Xie J: Structure and function of mycobacterium glycopeptidolipids from comparative genomics perspective. J Cell Biochem 2013, 114(8):1705-1713.

49. Lipworth S, Hough N, Leach L, Morgan M, Jeffery K, Andersson M, Robinson E, Smith EG, Crook D, Peto T et al: Whole-Genome Sequencing for Predicting Clarithromycin Resistance in Mycobacterium abscessus. Antimicrob Agents Chemother 2019, 63(1).

50. Ripoll F, Deshayes C, Pasek S, Laval F, Beretti JL, Biet F, Risler JL, Daffe M, Etienne G, Gaillard $\mathrm{JL}$ et al: Genomics of glycopeptidolipid biosynthesis in Mycobacterium abscessus and $\mathbf{M}$. chelonae. BMC Genomics 2007, 8:114.

51. Davidson RM: A Closer Look at the Genomic Variation of Geographically Diverse Mycobacterium abscessus Clones That Cause Human Infection and Disease. Front Microbiol 2018, 9:2988.

52. Wetzstein N, Kohl TA, Schultze TG, Andres S, Bellinghausen C, Hügel C, Kempf VAJ, Lehn A, Hogardt M, Serve $\mathrm{H}$ et al: Antimicrobial Susceptibility and Phylogenetic Relations in a German Cohort Infected with Mycobacterium abscessus. J Clin Microbiol 2020, 58(12).

53. Tortoli E, Kohl TA, Trovato A, Baldan R, Campana S, Cariani L, Colombo C, Costa D, Cristadoro $S$, Di Serio MC et al: Mycobacterium abscessus in patients with cystic fibrosis: low impact of inter-human transmission in Italy. Eur Respir J 2017, 50(1).

54. Schwarz C, Hogardt M: Empfehlungen an CF-Ambulanzen zur Risikominimierung einer möglichen Übertragung von

nicht-tuberkulösen Mykobakterien (z. B. Mycobacterium abscessus). Epidemiologisches Bulletin 2014, 13:107-107. 
513 55. Shoulah SA, Oschmann AM, Selim A, Semmler T, Schwarz C, Kamal E, Hamouda F, Galila E, Bitter W, Lewin A: Environmental Mycobacterium avium subsp. hominissuis have a higher

probability to act as a recipient in conjugation than clinical strains. Plasmid 2018, 95:28-35.

56. Rüger K, Hampel A, Billig S, Rücker N, Suerbaum S, Bange FC: Characterization of rough and smooth morphotypes of Mycobacterium abscessus isolates from clinical specimens. J Clin Microbiol 2014, 52(1):244-250.

57. Lavollay M, Dubée V, Heym B, Herrmann JL, Gaillard JL, Gutmann L, Arthur M, Mainardi JL: In vitro activity of cefoxitin and imipenem against Mycobacterium abscessus complex. Clin Microbiol Infect 2014, 20(5):0297-300.

58. Wallace Jr RJ, Meier A, Brown BA, Zhang Y, Sander P, Onyi GO, Böttger EC: Genetic basis for clarithromycin resistance among isolates of Mycobacterium chelonae and Mycobacterium abscessus. Antimicrobial Agents and Chemotherapy 1996, 40(7):1676-1681.

59. Smith DJ, Anderson GJ, Bell SC, Reid DW: Elevated metal concentrations in the CF airway correlate with cellular injury and disease severity. Journal of cystic fibrosis : official journal of the European Cystic Fibrosis Society 2014, 13(3):289-295.

60. Ranjan S, Yellaboina S, Ranjan A: IdeR in mycobacteria: from target recognition to physiological function. Crit Rev Microbiol 2006, 32(2):69-75.

61. Fabrino DL, Bleck CK, Anes E, Hasilik A, Melo RC, Niederweis M, Griffiths G, Gutierrez MG: Porins facilitate nitric oxide-mediated killing of mycobacteria. Microbes Infect 2009, 11(1011):868-875.

62. Ghazaei C: Mycobacterium tuberculosis and lipids: Insights into molecular mechanisms from persistence to virulence. Journal of Research in Medical Sciences 2018, 23(1).

63. Kreutzfeldt KM, McAdam PR, Claxton P, Holmes A, Seagar AL, Laurenson IF, Fitzgerald JR: Molecular Longitudinal Tracking of Mycobacterium abscessus spp. during Chronic Infection of the Human Lung. PLOS ONE 2013, 8(5). 


\section{Table captions:}

541 Table 1:

542 nsSNVs and larger deletions in 30 isolates from 11 serial samples obtained over 4.5 years from a

543 chronically infected patient. Shown are the locus tags of the homologous genes in the reference strain

544 ATCC 19977, the locus tags in the completed genome from the first sample (09-13-3), gene names,

545 annotations, functional COG categories and names of the isolates exhibiting the mutations when

546 compared to the initial isolate 09-13-3. Known associations to virulence and resistance of the genes or

547 homologous genes in other mycobacterial species are indicated in the last two columns.

548

549 Table 2:

550 Enrichment of PFAM protein domains in the pool of genes displaying non-synonymous mutations in 551 the M. abscessus isolates from patient $\mathrm{C}$ according to String network analysis. 


\section{$552 \quad$ Figure legends}

553 Fig. 1:

554 Phandango illustration showing a core genome-based Maximum likelihood tree corrected for

555 recombination events of $88 \mathrm{M}$. abscessus isolates from $11 \mathrm{CF}$ patients and the accessory genome. On

556 the left side the Maximum likelihood tree is shown, on the right-side gene presence in the respective

557 isolates is indicated by blue lines. Patients providing samples are named by letters (A to $S$ ). Names of

558 MABS isolates are composed as follows: first letter stands for patient, first number for the sample

559 number, second number for the year of isolation, last number for the colony number. Reference strains

560 included in the tree were DSM 44196 (= ATCC 19977) for M. abscessus abscessus (accession

561 NC_010397.1), CIP 108541 for M. abscessus bolletii (accession NZ_JRMF00000000.1) and

562 FLAC047 for M. abscessus massiliense (accession NZ_CP021122-1). Representatives of the global

563 patient-transmissible clusters [BIR 948 (accession ERS383065), RVI21 (accession ERS244779),

564 BIR1034 (accession ERS383155)] described in [6] were included to identify global cluster strains

565 present in the collection of CF isolates.

\section{Figure 2:}

567 Chronology of occurrence of non-synonymous mutations in M. abscessus isolated during 4.5 years

568 from patient $\mathrm{C}$. The different isolates are represented as coloured open circles below the time axis.

569 Filled circles in the lines below indicate that in this specific isolate the gene specified on the left has a

570 non-synonymous mutation. Filled circles in the last line (Plasmid) indicate loss of the plasmid. Only in

571 one isolate (70-16-1, $1^{\text {st }}$ isolate isolated after 38 months) the plasmid was not completely lost, but

572 partly deleted.

573 Figure 3:

574 Comparison of MICs from smooth and rough isolates from patient C. Nine smooth and nine rough $M$.

575 abscessus isolates from identical eight serial sputum samples were tested using the Sensititre system

576 (TREK diagnostics system, ThermoFisher Scientific). Out of the 13 antibiotics available in the

577 sensititre panel four (Amikacin, Cefoxitin, Imipenem and Linezolid) showed statistically significant

578 differences in MIC between the smooth and rough isolates from this patient (Mann Whitney Test).

579 Bars indicate the median values. 


\section{Legends for Supplementary Figures:}

582 Supplementary Figure S1:

583 Pan-genome-based maximum likelihood tree of $88 \mathrm{M}$. abscessus isolates from $11 \mathrm{CF}$ patients and

584 reference strains. Patients are named by letters (A to S). Names of M. abscessus isolates are composed

585 as follows: first letter stands for patient, first number for the year of isolation, last number for the

586 colony number. Reference strains included in the tree were DSM 44196 (= ATCC 19977) for $M$.

587 abscessus abscessus (accession NC_010397.1), CIP 108541 for M. abscessus bolletii (accession

588 NZ_JRMF00000000) and FLAC047 for M. abscessus massiliense (accession NZ_CP021122-1).

589 Representatives of the global patient-transmissible clusters [BIR 948 (accession ERS383065), RVI21

590 (accession ERS244779), BIR1034 (accession ERS383155)] described by [1] were included to identify

591 global cluster strains present in the collection of CF isolates. The scale of the bar represents the

592 percentage of substitutions per site.

593 Supplementary Fig. S2:

594 Plasmid map with annotations of plasmid pMabs-09-13 from isolate 09-13-3.

595 Supplementary Fig. S3:

596 Thin layer chromatography (TLC) of extracted glycopeptidolipids (GPL) from smooth and rough $M$.

597 abscessus isolates. GPL were extracted from strains 58-15-4 (A), 40-14-3 (B), 74-16-3 (C) and 65-16-

59817 (D) and subjected to TLC as described in Fujiwara et al. [PLoS One 2015, 10(5):e0126813]. Isolate

599 58-15-4 had a mutation mps2, isolate 40-14-3 in eryA, isolate 74-16-3 in mpsl, mtfD and MAB_4103c

600 and isolate 65-16-17 in mps 1 and MAB_4690c (Table 2). The different mutants displaying a rough

601 phenotype showed deviating GPL patterns when compared to the wildtype (E, isolate 23-13-1). 


\section{Captions of Supplementary Tables:}

604 Supplementary Table S1:

605 Patient characteristics and NTM isolation.

606 Supplementary Table S2:

607 Primers used for identification of NTM.

608 Supplementary Table S3:

609 List of isolates used for whole genome sequencing with accession numbers.

610 Supplementary Table S4:

611 Statistics for genome sequence assembly for isolate MABS 09-13-3 (MinION, accession

612 ERS4791737).

613

614 


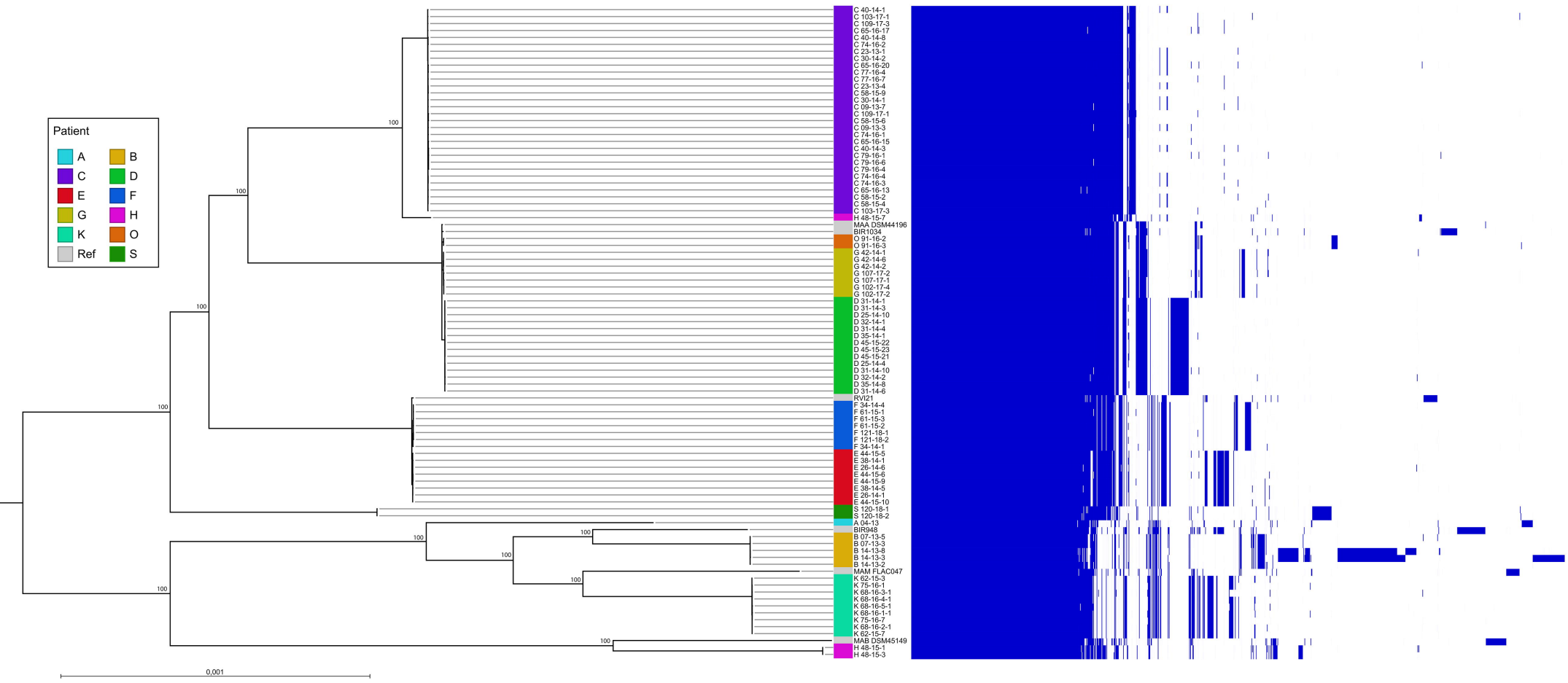




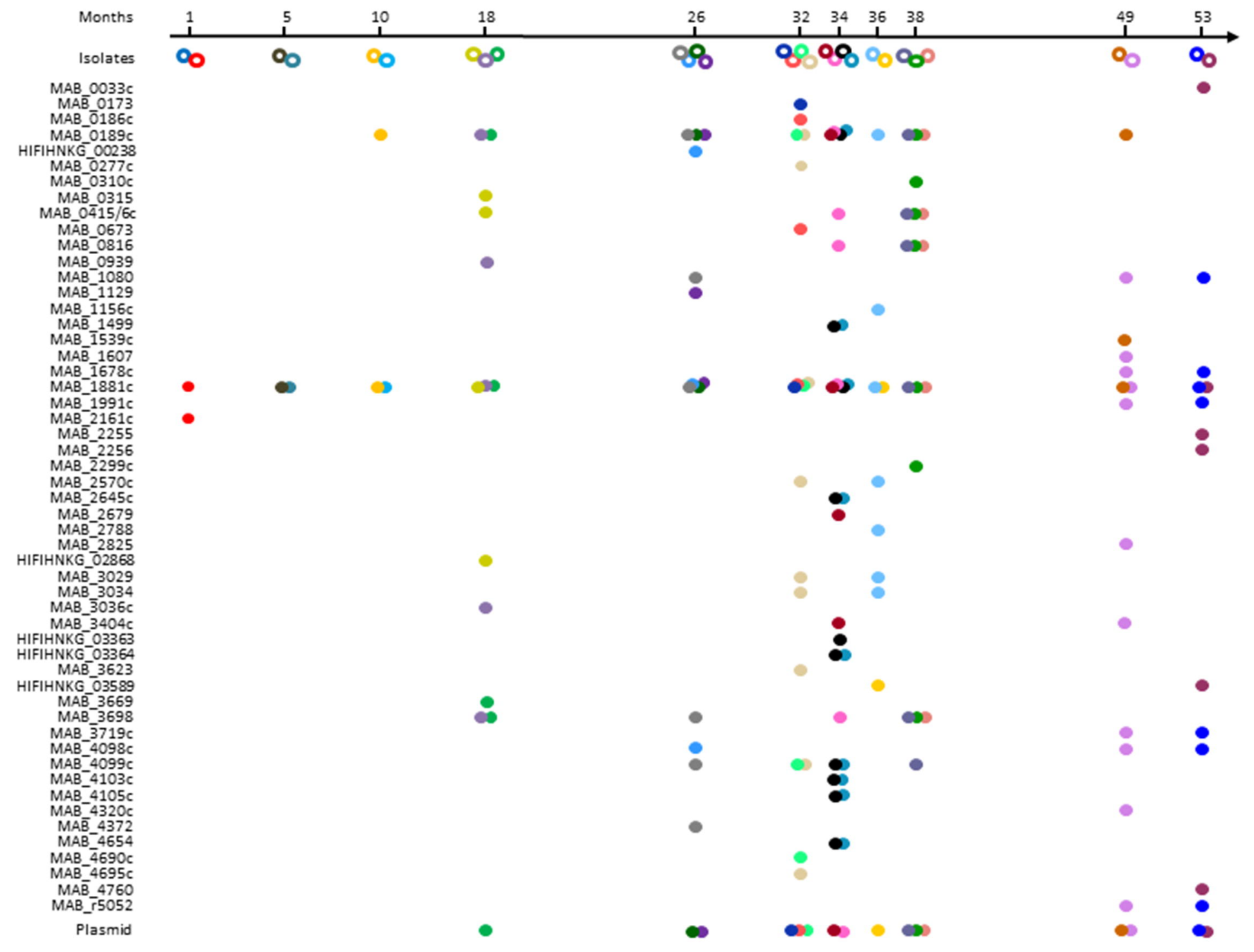


Amikacin

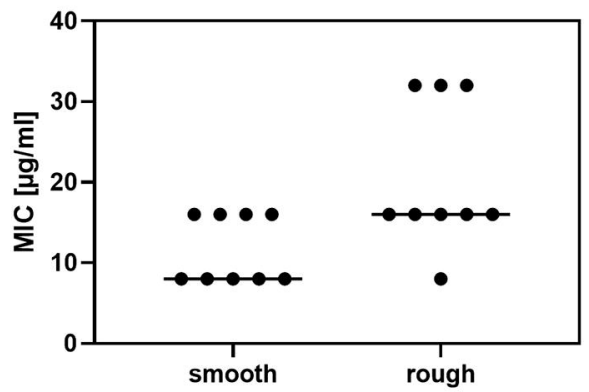

Imipenem

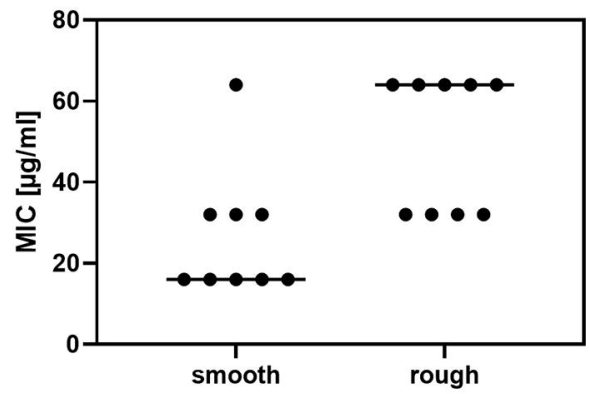

Cefoxitin

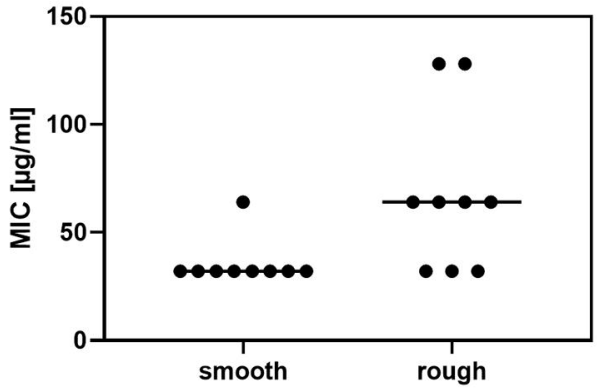

Linezolid

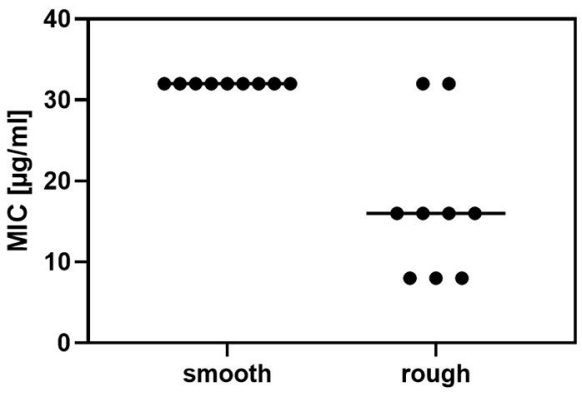

